\title{
Shared and Distinct Neurocognitive Endophenotypes of Schizophrenia and Psychotic Bipolar Disorder
}

\author{
Dohoon Kim, Jiwoo Kim, Taehoon Koo, Hyerim Yun, Seunghee Won \\ Department of Psychiatry, Kyungpook National University School of Medicine, Daegu, Korea
}

\begin{abstract}
Objective: Schizophrenia and bipolar disorder are characterized by the presence of neurocognitive impairments on the psychosis continuum. The present study aimed to explore the shared and distinct endophenotypes between these disorders. Methods: The study included 34 probands with remitted schizophrenia and 34 probands with euthymic bipolar disorder who had a history of psychotic symptoms that met the Diagnostic and Statistical Manual of Mental Disorders 4th edition (DSM-IV) criteria, unaffected first-degree relatives of probands (31 relatives of probands with schizophrenia and 29 relatives of probands with bipolar disorder), and 34 healthy controls. Cognitive assessments were performed using the digit span, continuous performance, Rey auditory and visual learning, complex figure, verbal fluency, Wisconsin card sorting, and finger tapping tests. Results: Probands with schizophrenia showed the most generalized and severe cognitive deficits across cognitive domains (working memory, verbal learning and memory, visual memory, verbal fluency, and executive function). Some domains of cognitive function (working memory, verbal learning, and memory) were also impaired in probands with bipolar disorder, but to a lesser degree than in probands with schizophrenia. All probands and relatives showed a common deficit in working memory compared to healthy controls. Relatives of probands with schizophrenia also showed verbal fluency dysfunction. Cognitive performance of all relatives was intermediate to the performance of both patients and healthy controls.

Conclusion: These findings suggest that a deficit in working memory could be a shared endophenotype of genetic vulnerability to schizophrenia and psychotic bipolar disorder, and verbal fluency could be a candidate endophenotype for schizophrenia specifically.
\end{abstract}

KEY WORDS: Schizophrenia; Bipolar disorder; Endophenotypes; Cognition.

\section{INTRODUCTION}

According to the "Kraepelinian dichotomy", schizophrenia (SZ) and bipolar disorder (BD) have been regarded as different nosologic categories. This conception has been maintained in the current versions of the diagnostic classifications for mental disorders. However, several issues challenge the dichotomous paradigm of psychotic disorders with respect to $\mathrm{SZ}$ and $\mathrm{BD} .{ }^{1)}$ These issues include the overlapping organic basis and blurred boundaries between psychoses, as indicated by emerging biological data ${ }^{2,3)}$; the non-specificity of psychopathological features, such as perceptual disturbances and affective symptoms, between $\mathrm{SZ}$ and $\mathrm{BD}$; and the shared genetic

\footnotetext{
Received: January 25, 2015 / Revised: March 18, 2015

Accepted: March 24, 2015

Address for correspondence: Seunghee Won, MD, PhD

Department of Psychiatry, Kyungpook National University Hospital,

130 Dongdeok-ro, Jung-gu, Daegu 700-721, Korea

Tel: +82-53-200-5747, Fax: +82-53-426-5361

E-mail:wonsh864@knu.ac.kr
}

susceptibility loci uncovered within recent genome-wide association studies. ${ }^{4,5}$ Because of these challenges, some researchers have suggested a dimensional approach to the psychosis continuum; the relative admixture of psychotic and affective symptoms would show a continuous distribution between $\mathrm{SZ}$ and psychotic BD. ${ }^{1)}$

Many researchers have tried to identify whether SZ and $\mathrm{BD}$ should be considered as separate diseases or different manifestations of the same pathophysiological process. Establishing similarities and differences in the biological markers of SZ and BD may provide important insights for future genetic studies, and may improve conceptualizations about the common and distinct aspects of the pathophysiology, about clinical heterogeneity, and about the clinical boundaries of the two disorders. To overcome these clinical limitations, many researchers have tried to find endophenotypes that are specific to each disorder. These intermediate phenotypes are disease-associated traits that more strongly indicate the underlying molecular genetic background than the clinical phenotype itself.

(c) This is an Open-Access article distributed under the terms of the Creative Commons Attribution Non-Commercial License (http://creativecommons.org/licenses/by-nc/3.0) which permits unrestricted non-commercial use, distribution, and reproduction in any medium, provided the original work is properly cited. 
Consistent with the suggested criteria for the identification of endophenotypic markers, allied phenotypes should be (a) associated with illness, (b) heritable, (c) state-independent, (d) co-segregated with illness within families, and (e) also found in unaffected relatives at a higher rate than in the general population. ${ }^{6}$ One of the widely studied putative endophenotypes in psychiatric syndromes is cognitive dysfunction. The established heritability of cognitive function and the neural networks mediating specific aspects of cognition, as well as the availability of objective measurement tools to assess cognitive performance provide support for examining cognitive function as a promising endophenotype for SZ and BD. ${ }^{7)}$

The literature reports that the generalized cognitive deficit in probands with SZ is present at the first episode of psychosis, relatively stable over time, largely independent of clinical status or antipsychotic treatment, and is an important cause of functional disability. Probands with BD also exhibit cognitive dysfunction even when in a euthymic state, and this tendency is preserved between mood episodes and is generally stable in chronic patients. Moreover, the cognitive impairments of BD can be strongly related to poor functional outcomes and present before illness onset. ${ }^{8)}$ A meta-analysis on direct comparison studies of cognitive dysfunction have reported that probands with BD tend to show generalized cognitive impairment, although it is less severe than that of probands with SZ. ${ }^{9)}$

As there is a high genetic influence on the development of $\mathrm{SZ}$ and $\mathrm{BD}$, unaffected relatives are also anticipated to show some cognitive dysfunction. In this context, many studies of first relatives of probands with SZ and BD have explored whether cognitive impairment qualifies as a good candidate for an endophenotype for SZ or BD. Some recent studies of probands with SZ and their relatives have proposed verbal memory, executive function, working memory, verbal fluency, and sustained attention as potential endophenotypes for SZ. ${ }^{10,11)}$ For BD, executive function, verbal memory, response inhibition, processing speed, and working memory were also regarded as candidates for endophenotypes. ${ }^{12-15)}$ Although cognitive impairments overlap in SZ and BD and may be a marker of genetic vulnerability for both disorders, only a small number of studies have directly compared neurocognitive performance in probands with $\mathrm{SZ}$, probands with $\mathrm{BD}$, and their relatives. The findings suggest that cognitive dysfunction as a marker of familial vulnerability is stronger for SZ than for BD but the specific cognitive domains relating to shared or distinct endophenotypes were unclear. ${ }^{16-20)}$ To overcome the methodological issues of the previous stud- ies, further detailed study is needed that directly compares cognitive functions in these disorders using identical testing and recruitment strategies, covers a broader range of cognitive domains, and maintains diagnostic homogeneity within the samples.

In this study, we directly compare the levels and patterns of neurocognitive function in probands with SZ, probands with psychotic BD, their unaffected first-degree relatives, and healthy community samples, strictly using the same neuropsychological battery. We aim to explore shared and distinct putative endophenotypic markers for SZ and psychotic BD.

\section{METHODS}

\section{Participants}

The cross-sectional study sample comprised 162 participants in total: 34 probands with SZ (SZ-P), 34 probands with BD having a history of psychotic symptoms (BD-P), 31 first-degree relatives of SZ-P (SZ-R), 29 first-degree relatives of BD-P (BD-R), and 34 healthy controls (HC). All participants gave their written informed consent to participate in the study after a detailed explanation of the study procedures.

The probands were recruited from the outpatient clinic of Kyungpook National University Hospital and had to meet the Diagnostic and Statistical Manual of Mental Disorders 4th edition $^{21)}$ (DSM-IV) criteria for SZ or BD. In the case of BD, all probands had bipolar I disorder with a history of psychotic symptoms. The relatives were first-degree biological relatives of probands without a personal history of psychiatric disorder. A complete family history of the first-degree relatives was obtained from each proband and from at least one other first-degree relative. The HC participants, in who there was an absence of a personal and family history of DSM-IV axis I or II disorders, were recruited through a local advertisement. The Korean version of the Structured Clinical Interview for DSM-IV Axis I Disorders ${ }^{22)}$ (SCID-I) was administered to all participants to confirm their diagnostic eligibility. All groups were matched on age, sex, and years of education. All participants had to be aged between 18 and 50 years. All probands were required to have been in remission at for least 3 months, with no change in medication or symptoms for at least 1 month, before cognitive evaluation and did not have their medication withdrawn for the purpose of the study. For inclusion in the study, all participants had to be normothymic, as evaluated by the Korean version of the Montgomery-Åsberg Depression Rating Scale ${ }^{23)}$ 
(K-MADRS) (score $<8$ ) and the Korean version of the Young Mania Rating Scale ${ }^{24)}$ (YMRS-K) (score <6), and not to be psychotic, as evaluated by the Brief Psychiatric Rating Scale $^{25)}$ (BPRS) (score $\left.\leq 30\right)$. Additional inclusion criteria for the participants were the absence of head trauma, neurologic disorders, alcohol or substance abuse, mental retardation (intelligence quotinent $[\mathrm{IQ}]<70$ ), and serious medical conditions. The institutional review boards of the Kyungpook National University Hospital approved this study (IRB number 2011-01-042).

\section{Neurocognitive Assessment}

Intellectual functioning was estimated ${ }^{26)}$ using two subtests (vocabulary, block design) of the Korean Wechsler Adult Intelligence Scale. ${ }^{27)}$ The following neurocognitive tests were conducted. The digit span test (DST) used in this study was composed of forward and backward subtests. The forward subtest evaluates the effectiveness of attention, whereas the backward subtest represents working memory more than attention. The continuous performance test ${ }^{28)}(\mathrm{CPT})$ is a computerized test assessing visual and auditory sustained and selective attention. The Korean Rey auditory and verbal learning test $^{29)}$ (K-AVLT) is a measure of verbal learning and memory. The Korean complex figure test ${ }^{29)}$ (K-CFT) allows the clinician to assess visuospatial abilities, planning, visual memory, and attention. The verbal fluency test $^{30)}$ (VFT) examines language production and retrieval function. The Wisconsin card sorting test ${ }^{31)}$ (WCST) was administered to measure executive function and cognitive flexibility. Finally, the finger tapping test ${ }^{32)}$ (FTT) was used to measure motor speed.

\section{Statistical Analyses}

All analyses were conducted using PASW Statistics ver. 18.0 for Windows software (IBM Co., Armonk, NY, USA). Values of $p<0.05$ were regarded as significant. For the demographic data, we performed analysis of variance (ANOVA) to examine group differences for age, education, BPRS score, K-MADRS score, and YMRS-K score. Following ANOVA, we used Dunnett's T3 post hoc correction to compare between groups. We used the chi-square test for gender analysis. The $t$-test was used to compare clinical characteristics between SZ-P and BD-P groups.

When analyzing the neurocognitive battery results, we performed ANOVA and the Bonferroni post hoc comparison, similar to the analysis of demographic data. Following this, analysis of covariance (ANCOVA) was performed with the BPRS, YMRS-K, and K-MADRS scores as covariates to control for group differences in the demographic data.

We evaluated the patterns of performance across tests (i.e., profiles) within the six neurocognitive domains. To do so, we converted each neurocognitive dependent variable into a z score using the mean and standard deviations for HC group. The values of each cognitive domain obtained from a significant subtests or composite scores of each cognitive tests; attention from forward subtest in DST; working memory from backward subtest in DST; verbal memory from immediate recall composite in K-AVLT; visual memory from immediate recall composite in K-CFT; verbal fluency from category composite in VFT; executive function from perseverative error in WCST.

\section{RESULTS}

\section{Demographics and Clinical Characteristics}

The demographic characteristics and clinical features are summarized in Table 1. The five groups of participants were comparable for age, gender, and education. The estimated total intelligence of the SZ-P group was lower than that of the other groups, but there was not a statistically significant difference $(F=2.4, p=0.051)$. Although psychopathologies in all groups were within the criteria, there were group differences. In the post hoc comparison, the BPRS mean score of the SZ-P group was significantly higher than that of any other group $(F=33.2, p<0.001)$. SZ-P group showed significantly higher K-MADRS scores than BD-R and HC groups $(F=3.2, p=0.014)$. BD-P group had significantly higher YMRS-K scores than BD-R and HC groups $(F=5.4, p<0.001)$. The $t$-tests showed no main effects of group for onset age, duration of illness, or duration of taking medication between SZ-P and BD-P groups. All of the patients were taking antipsychotics at the time of the study.

\section{Neurocognitive Function}

The results of the neurocognitive tests (presented in Table 2) for each cognitive domain are as follows.

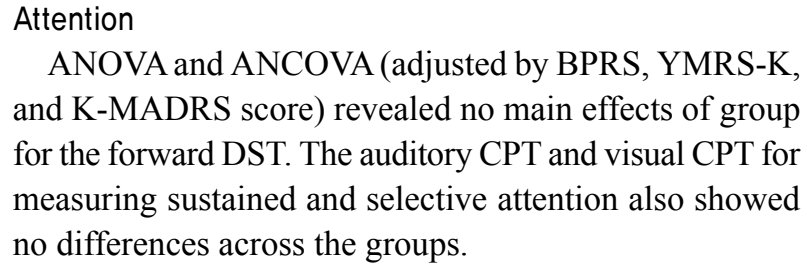
and K-MADRS score) revealed no main effects of group for the forward DST. The auditory CPT and visual CPT for measuring sustained and selective attention also showed no differences across the groups. 
Table 1. Demographic and clinical characteristics of each group

\begin{tabular}{|c|c|c|c|c|c|c|c|c|}
\hline \multirow[b]{2}{*}{ Characteristic } & \multicolumn{5}{|c|}{ Group } & \multicolumn{3}{|c|}{ Statistics } \\
\hline & $\begin{array}{l}\text { SZ-P (a) } \\
(n=34)\end{array}$ & $\begin{array}{c}\text { BD-P (b) } \\
(n=34)\end{array}$ & $\begin{array}{l}\text { SZ-R (c) } \\
(n=31)\end{array}$ & $\begin{array}{c}\text { BD-R (d) } \\
(n=29)\end{array}$ & $\begin{array}{l}H C(e) \\
(n=34)\end{array}$ & F or $t$ & $p$ value & Post-hoc* \\
\hline Sex, female & $17(50.0)$ & $23(67.6)$ & $20(64.5)$ & $17(58.6)$ & $17(50.0)$ & & $0.458^{\dagger}$ & \\
\hline Age $(y r)$ & $31.2 \pm 7.7$ & $31.4 \pm 7.5$ & $31.3 \pm 10.1$ & $31.8 \pm 8.0$ & $30.5 \pm 8.1$ & 0.1 & 0.982 & \\
\hline Education (yr) & $14.2 \pm 2.1$ & $14.1 \pm 1.6$ & $14.0 \pm 1.6$ & $13.9 \pm 2.2$ & $14.7 \pm 1.4$ & 1 & 0.370 & \\
\hline Age of onset (yr) & $24.3 \pm 6.8$ & $24.9 \pm 6.2$ & - & - & - & -0.3 & 0.691 & \\
\hline Duration of illness (yr) & $7.1 \pm 6.3$ & $6.1 \pm 4.2$ & - & - & - & 0.7 & 0.477 & \\
\hline Duration of current remission (mo) & $18.5 \pm 18.1$ & $9.7 \pm 9.6$ & - & - & - & 2.1 & 0.033 & \\
\hline Admission (n) & $1.1 \pm 1.4$ & $2.6 \pm 2.2$ & - & - & - & -3.3 & 0.001 & \\
\hline \multicolumn{9}{|l|}{ Mood episode (n) } \\
\hline Manic episode & - & $31 \pm 2.6$ & - & - & - & & & \\
\hline Depressive episode & - & $23 \pm 3.2$ & - & - & - & & & \\
\hline Mixed episode & - & $26 \pm 0.2$ & - & - & - & & & \\
\hline Total episode & - & $24 \pm 5.8$ & - & - & - & & & \\
\hline \multicolumn{9}{|l|}{ Psychopathology } \\
\hline Total $\mid Q$, estimate & $107.3 \pm 13.4$ & $108.6 \pm 11.2$ & $110.2 \pm 13.6$ & $107.2 \pm 12.3$ & $115.2 \pm 11.0$ & 2.4 & 0.051 & \\
\hline BPRS (score) & $20.4 \pm 3.8$ & $16.1 \pm 2.2$ & $15.2 \pm 2.3$ & $14.8 \pm 1.6$ & $14.4 \pm 1.1$ & 33.2 & $<0.001$ & $a>b, c, d, e$ \\
\hline YMRS-K (score) & $0.9 \pm 1.3$ & $1.3 \pm 1.7$ & $0.5 \pm 1.1$ & $0.2 \pm 0.4$ & $0.2 \pm 0.4$ & 5.4 & $<0.001$ & $b>d, e$ \\
\hline K-MADRS (score) & $3.4 \pm 2.7$ & $2.5 \pm 2.7$ & $2.5 \pm 2.9$ & $1.3 \pm 2.0$ & $1.6 \pm 2.2$ & 3.2 & 0.014 & $a>d, e$ \\
\hline Duration of taking medication (yr) & $5.9 \pm 6.3$ & $5.8 \pm 4.9$ & - & - & - & 0.7 & 0.944 & \\
\hline \multicolumn{9}{|l|}{ Medication status } \\
\hline Atypical antipsychotics & $33(97.1)$ & $27(79.4)$ & - & - & - & & & \\
\hline Anticonvulsants & $0(0.0)$ & $25(73.5)$ & - & - & - & & & \\
\hline Lithium & $0(0.0)$ & $3(8.8)$ & - & - & - & & & \\
\hline Antidepressants & $3(8.8)$ & $5(14.7)$ & - & - & - & & & \\
\hline Benzodiazepine & $14(41.2)$ & $13(38.2)$ & - & - & - & & & \\
\hline Anticholinergics & $9(26.5)$ & $0(0.0)$ & - & - & - & & & \\
\hline
\end{tabular}

Values are presented as mean \pm standard deviation or number (\%)

SZ-P, schizophrenia patients; BD-P, bipolar I disorder patients; SZ-R, unaffected first-degree relatives of SZ-P; BD-R, unaffected first-degree relatives of $\mathrm{BD}-\mathrm{P} ; \mathrm{HC}$, healthy controls; $1 \mathrm{Q}$, intelligence quotinent; BPRS, Brief Psychiatric Rating Scale; YMRS-K, Korean Version of Young Mania Rating Scale; K-MADRS, Korean Version Montgomery-Åsberg Depression Rating Scale.

Analysis of variance (ANOVA) was used; *by Dunnett T3 test, ${ }^{\dagger}$ by chi square test.

\section{Working memory}

The backward DST scores of SZ-P, BD-P, SZ-R, and BD-R groups were significantly different from those of HC group $(F=5.042, p=0.001)$.

\section{Verbal learning and memory}

Learning performance on the K-AVLT for verbal learning showed significant differences between the SZ-P group and four other groups $(F=12.626, p=0.000)$. The $\mathrm{BD}-\mathrm{P}$ group was worse than the $\mathrm{HC}$ group but better than the SZ-P group $(F=12.626, p=0.000)$. There was a difference between SZ-P group and both relatives and HC groups in immediate and delayed recall on the K-AVLT for verbal memory $(F=7.8222, p=0.000 ; F=9.536, p=0.000$, respectively). BD-P group performed significantly better than SZ-R group in immediate recall $(F=7.822, p=0.000)$ and than SZ-R and HC groups in delayed recall $(F=9.536$, $p=0.000)$.

\section{Visual learning and memory}

Compared with the HC group, the SZ-P group showed significant impairments in immediate $(F=3.399, p=0.011)$ and delayed recall $(F=3.601, p=0008)$. This reflects deficits in visual memory. There were no group differences in the copy scores on the K-CFT for visual learning.

\section{Verbal fluency}

In the category subtest of VFT, significant differences were found between the SZ-P group and the four other groups $(F=10.317, p=0.000)$. The SZ-R group also performed significantly worse than the HC group $(F=12.626$, $p=0.000)$. In the lexicon subtest of the VFT, the SZ-P group was significantly worse than the HC group $(F=4.033, p=0.004)$.

\section{Executive function}

In the WCST, there was a group difference in perseverative error (which measures cognitive flexibility). The SZ-P group showed significantly poorer performance 
Table 2. Group differences in neurocognitive tests of each group

\begin{tabular}{|c|c|c|c|c|c|c|c|c|c|}
\hline \multirow[b]{2}{*}{ Variable } & \multicolumn{5}{|c|}{ Group } & \multicolumn{4}{|c|}{ Statistics } \\
\hline & $\begin{array}{l}\text { SZ-P (a) } \\
(n=34)\end{array}$ & $\begin{array}{c}\text { BD-P (b) } \\
(n=34)\end{array}$ & $\begin{array}{c}\text { SZ-R (c) } \\
(n=31)\end{array}$ & $\begin{array}{c}\text { BD-R (d) } \\
(n=29)\end{array}$ & $\begin{array}{l}\mathrm{HC}(e) \\
(n=34)\end{array}$ & $F$ & $p$ value & Post-hoc* & ANCOVA \\
\hline \multicolumn{10}{|l|}{ Digit span test } \\
\hline Forward span & $7.4 \pm 1.1$ & $7.1 \pm 1.2$ & $7.5 \pm 1.5$ & $7.4 \pm 1.3$ & $7.8 \pm 1.1$ & 1.231 & 0.300 & & 0.481 \\
\hline Backward span & $4.5 \pm 1.3$ & $5.0 \pm 1.3$ & $4.9 \pm 1.2$ & $4.9 \pm 1.6$ & $6.0 \pm 1.6$ & 5.042 & 0.001 & $e>a, b, c, d$ & 0.003 \\
\hline \multicolumn{10}{|l|}{ Auditory CPT } \\
\hline Correct response & $49.1 \pm 16.0$ & $51.7 \pm 15.8$ & $56.1 \pm 17.5$ & $52.0 \pm 13.7$ & $56.0 \pm 15.0$ & 1.241 & 0.296 & & 0.566 \\
\hline Omission errors & $49.1 \pm 16.0$ & $51.7 \pm 15.8$ & $56.1 \pm 17.5$ & $52.0 \pm 13.7$ & $56.0 \pm 15.0$ & 1.241 & 0.296 & & 0.566 \\
\hline Commission errors & $47.2 \pm 14.5$ & $47.8 \pm 16.3$ & $48.5 \pm 15.9$ & $52.8 \pm 14.1$ & $52.0 \pm 14.0$ & 0.921 & 0.454 & & 0.407 \\
\hline SDRT & $45.4 \pm 9.9$ & $45.4 \pm 9.5$ & $45.1 \pm 11.6$ & $46.2 \pm 7.5$ & $50.9 \pm 10.9$ & 1.993 & 0.098 & & 0.211 \\
\hline \multicolumn{10}{|l|}{ Visual CPT } \\
\hline Correct response & $58.8 \pm 17.8$ & $61.2 \pm 18.5$ & $63.1 \pm 16.0$ & $62.5 \pm 16.9$ & $64.6 \pm 16.4$ & 0.547 & 0.702 & & 0.898 \\
\hline Omission errors & $58.8 \pm 17.8$ & $61.2 \pm 18.5$ & $63.1 \pm 16.0$ & $62.5 \pm 16.9$ & $64.6 \pm 16.4$ & 0.547 & 0.702 & & 0.898 \\
\hline Commission errors & $61.3 \pm 18.5$ & $60.0 \pm 18.7$ & $64.0 \pm 17.1$ & $69.3 \pm 16.4$ & $64.9 \pm 16.4$ & 1.410 & 0.233 & & 0.233 \\
\hline SDRT & $49.6 \pm 9.1$ & $50.6 \pm 11.1$ & $50.6 \pm 14.3$ & $51.5 \pm 11.0$ & $55.9 \pm 9.3$ & 1.702 & 0.152 & & 0.470 \\
\hline \multicolumn{10}{|l|}{ K-AVLLT } \\
\hline Learning (trial 1-5) & $38.9 \pm 9.4$ & $46.5 \pm 9.8$ & $49.5 \pm 7.7$ & $50.1 \pm 8.2$ & $52.8 \pm 8.1$ & 12.626 & 0.000 & $b, c, d, e>a \& e>b>a$ & 0.000 \\
\hline Immediate recall & $8.1 \pm 3.3$ & $9.5 \pm 2.0$ & $11.3 \pm 2.0$ & $10.5 \pm 2.3$ & $10.2 \pm 2.3$ & & 0.000 & $c, d, e>a \& c>b$ & 0.008 \\
\hline Delayed recall & $7.7 \pm 3.0$ & $9.0 \pm 3.2$ & $11.4 \pm 2.8$ & $10.5 \pm 2.5$ & $11.2 \pm 2.9$ & $\begin{array}{l}7.822 \\
9.536\end{array}$ & 0.000 & $c, d, e>a \& c, e>b$ & 0.002 \\
\hline \multicolumn{10}{|l|}{$\mathrm{K}-\mathrm{CFT}$} \\
\hline Copy & $11.9 \pm 2.2$ & $12.4 \pm 2.4$ & $12.0 \pm 2.5$ & $12.4 \pm 2.6$ & $12.8 \pm 2.2$ & 0.734 & 0.570 & & 0.477 \\
\hline Immediate recall & $8.2 \pm 3.1$ & $9.6 \pm 3.4$ & $10.4 \pm 3.2$ & $9.6 \pm 3.9$ & $11.2 \pm 3.1$ & 3.399 & 0.011 & $e>a$ & 0.009 \\
\hline Delayed recall & $8.2 \pm 3.3$ & $9.5 \pm 3.7$ & $10.3 \pm 3.3$ & $9.8 \pm 3.7$ & $11.3 \pm 3.3$ & 3.601 & 0.008 & $e>a$ & 0.009 \\
\hline \multicolumn{10}{|l|}{ Verbal fluency } \\
\hline Category & $29.3 \pm 6.2$ & $37.4 \pm 9.0$ & $35.6 \pm 6.4$ & $37.8 \pm 8.9$ & $42.0 \pm 10.2$ & 10.317 & 0.000 & $b, c, d, e>a \& e>c$ & 0.000 \\
\hline \multirow[t]{2}{*}{ Lexicon } & $30.6 \pm 10.6$ & $37.3 \pm 10.8$ & $35.9 \pm 12.3$ & $34.2 \pm 10.5$ & $41 \pm 11.0$ & & 0.004 & $e>a$ & 0.041 \\
\hline & & & & & & 4.033 & & & \\
\hline \multicolumn{10}{|l|}{ WCST } \\
\hline Categories completed & $5.3 \pm 1.4$ & $5.7 \pm 0.9$ & $5.7 \pm 0.9$ & $5.8 \pm 0.5$ & $5.7 \pm 0.8$ & 1.254 & 0.291 & & 0.644 \\
\hline Perseverative error & $50.2 \pm 7.4$ & $55.3 \pm 8.1$ & $55.3 \pm 8.0$ & $55.9 \pm 8.9$ & $57.1 \pm 9.7$ & 3.223 & 0.014 & $e>a$ & 0.045 \\
\hline \multicolumn{10}{|l|}{ Finger tapping } \\
\hline Dominant hand & $59.4 \pm 12.9$ & $63.0 \pm 12.1$ & $67.4 \pm 8.9$ & $66.5 \pm 11.7$ & $64.3 \pm 11.9$ & 2.143 & 0.078 & & 0.114 \\
\hline Non-dominant hand & $61.9 \pm 12.0$ & $64.6 \pm 12.8$ & $66.3 \pm 11.5$ & $65.6 \pm 10.7$ & $66.4 \pm 13.0$ & 0.732 & 0.572 & & 0.818 \\
\hline
\end{tabular}

Values are presented as mean \pm standard deviation (SD).

SZ-P, schizophrenia patients; BD-P, bipolar I disorder patients; SZ-R, unaffected first-degree relatives of SZ-P; BD-R, unaffected first-degree relatives of BD-P; HC, healthy controls; CPT, continuous performance test; SDRT, SD of response time; K-AVLT, Korean version of auditory verbal learning test; K-CFT, Korean version of complex figure test; WCST, Wisconsin card sorting test.

ANCOVA (BPRS, K-MADRS, YMRS-K score as covariates) was used; *by Bonferroni test.

than the HC group $(F=3.223, p=0.014)$.

\section{Motor speed}

There were no group differences in the FTT.

\section{Patterns of neurocognitive function across groups}

The profile graphs for the neurocognitive domains are presented in Fig. 1. The SZ-P group had a different profile from the other four groups that appeared to be due to relatively smaller group differences in attention, working memory, and executive function, and larger group differences in verbal memory and verbal fluency. The profile for BD-P group overlapped with that for SZ-P group, and BD-P group showed less cognitive impairments than SZ-P group. The cognitive performance of both types of relative was intermediate to the performance of both types of probands and $\mathrm{HC}$ groups. Both types of relatives showed similar performance profiles.

\section{DISCUSSION}

The present study explored which cognitive domain may serve as a shared endophenotype or may be a specific endophenotype for $\mathrm{SZ}$ and psychotic BD. Our results indicate that working memory deficit might be the most prominent candidate for a shared endophenotype between $\mathrm{SZ}$ and psychotic BD. In addition, dysfunction of verbal fluency was suggested as a potential endophenotypic marker specifically for SZ.

In the present study, SZ-P exhibited a generalized cognitive impairment with pronounced deficits in the domains of working memory, verbal learning and memory, 


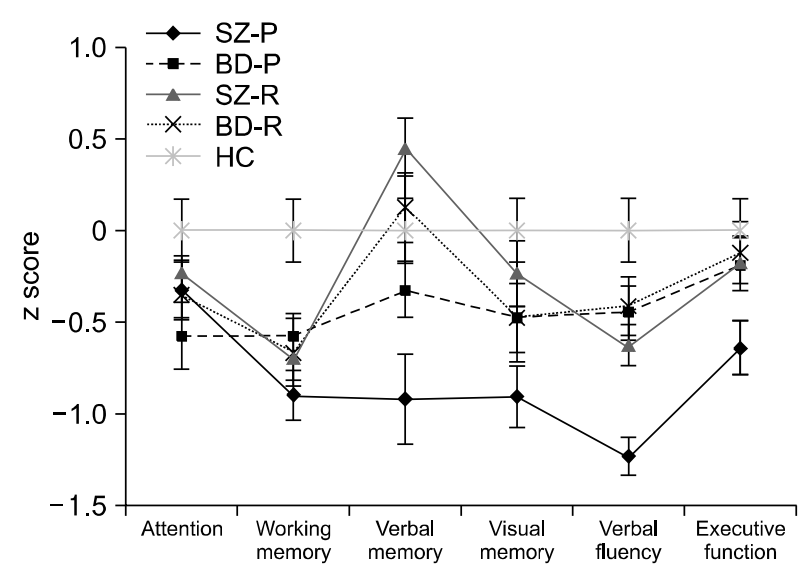

Fig. 1. Group differences in neuropsychological profiles of schizophrenia patients (SZ-P), bipolar I disorder patients (BD-P), unaffected first-degree relatives (SZ-R, BD-R) and healthy controls $(H C)$. The values of each cognitive domain obtained from a significant subtests or composite scores of each cognitive tests; attention from forward subtest in digit span test; working memory from backward subtest in digit span test; verbal memory from immediate recall composite in auditory verbal learning test; visual memory from immediate recall composite in complex figure test; verbal fluency from category composite in verbal fluency test; executive function from perseverative error in Wiscosin card sorting test. The $y$-axis indicates $z$ scores converted using the mean and standard deviation of the healthy controls. The values are presented as means with standard errors.

visual memory, verbal fluency, and executive function, but not attention. SZ-R showed deficits in working memory and verbal fluency. This indicates that working memory and verbal fluency are potential endophenotypes for SZ. Our finding of deficits in multiple cognitive domains in SZ-P is comparable with many previous studies that reported cognitive impairments across a broad range of neuropsychological domains but most consistently for the domains of memory, executive function, attention, and language function. ${ }^{33,34)}$ The finding that SZ-R performed intermediate to SZ-P and HC is in accordance with previous studies. ${ }^{10,11)}$ A meta-analysis of studies on SZ-R proposed several cognitive domains (verbal memory, executive function, working memory, verbal fluency, and sustained attention) as potential endophenotypes for SZ. ${ }^{10,11)}$ It is noteworthy that our results suggest that verbal fluency could be a strong candidate for an endophenotype for SZ, which is not shared with BD. This is confirmed by a meta-analysis of longitudinal studies of cognition that reported that, although a wide range of cognitive deficits have been demonstrated in SZ, semantic verbal fluency, which was stable in probands and showed least improvement over time by intervention, may represent the best promising endophenotype for $\mathrm{SZ}{ }^{34}$ )
Cognitive deficits in probands with BD and their relatives have been reported less consistently and some describe them as being state-dependent. Growing evidence suggests that BD may have distinct cognitive profiles depending on the presence of lifetime psychosis. That is, probands with psychotic BD show deficits in domains of verbal and non-verbal declarative memory, working memory, processing speed, executive function, and attention, which is similar to those found in SZ. ${ }^{35-37)}$ In addition, deficits in processing speed, working memory, declarative memory, and executive function have been reported in BD-R. The deficits in attention were less consistent. ${ }^{12-15,38)}$ Although some deficits were not observed in this study, BD-P had selective cognitive impairments, with pronounced deficits in the domains of working memory, and verbal learning and memory. BD-R presented only working memory deficits. The present study suggests that working memory could be an endophenotype for BD.

The present study also showed that the degree of cognitive deficits in BD-P was less severe than in SZ-P, and the profiles of the deficits were similar. An interesting finding was that the cognitive performance of both relatives was intermediate to the performance of both probands and HC. SZ-R (working memory and verbal fluency) were more cognitively impaired than BP-R (working memory). These findings also support the notion that psychosis may be a key clinical continuum associated with overlapping cognitive characteristics and may be a marker of genetic vulnerability for SZ and BD. ${ }^{8,18,19)}$

Another noteworthy finding was that working memory is a shared putative endophenotype between SZ and BD. Working memory is a limited-capacity storage system that involves the online maintenance and adjustment of information important for daily functioning. ${ }^{39)}$ In SZ, working memory deficits are found consistently across a range of tests. ${ }^{8)}$ They show little correlation with the severity of psychotic symptoms, ${ }^{40)}$ can be found in probands with remitted SZ, ${ }^{9}$ and seem to be relatively stable across time. ${ }^{41)}$ Working memory has been shown to have a moderately high level of heritability in a SZ sample. ${ }^{42)}$ Working memory deficits were apparent in studies of relatives of patients with SZ and showed a moderate effect size in previous meta-analyses. ${ }^{9}$ Working memory deficits were also reported in recent meta-analyses of cognitive impairment in probands with BD. ${ }^{43)}$ Although there were discrepancies between the meta-analyses of relatives of probands with $\mathrm{BD}$, some research has reported working memory to be one of the most powerful endophenotypes for BD. ${ }^{12,13)}$ The underlying neurobiological mechanisms 
of working memory impairments also support our research. In the normal population, the activation of working memory is related to activation of the dorsolateral prefrontal cortex and activation of the inferior and posterior parietal cortex. ${ }^{44)}$ Aberrant activation of the prefrontal cortex during a working memory task is commonly observed in probands with $\mathrm{SZ}^{45)}$ and their first-degree relatives $^{46)}$ and in probands with $\mathrm{BD}^{47)}$ and their first-degree relatives. $^{48)}$ Recently, two literature reviews comparing cognitive impairment between SZ and BD reported that deficits in some cognitive functions, such as working memory, episodic memory, cognitive control, attention regulation, and emotional processing, offer potential for identifying shared and illness-specific allied neurocognitive phenotypes for SZ and BD. ${ }^{8,49)}$ Further, Ivleva et $a l .{ }^{19)}$ reported that impairments in several cognitive functions (working and declarative memory, executive function, and attention) were observed concurrently in recruited probands with $\mathrm{SZ}$ and psychotic $\mathrm{BD}$, along with their biological relatives.

Contrary to what we expected, there were no group differences in the attention domain (including selective and sustained attention). Deficits in attention of all probands and relatives of SZ are reported in previous research. ${ }^{8)}$ Our results are generally consistent with most previous studies. However, there were some discrepancies with previous studies in selected cognitive domains, which can be explained partly by the characteristics of our sample. First, the progress of both diseases had not yet reached an advanced stage, the mean age of the probands was relatively lower, the age of disease onset was not as early, the duration of illness was not as long, the mean frequency of admission was 1-2 times, and the duration of remission was longer. Hill et al. ${ }^{20)}$ reported that the severity of cognitive impairments across psychotic disorders was consistent with prominent affective features, and less enduring psychosis was associated with less cognitive impairment. Second, the participants, including the probands, were matched for IQ, age, gender, and education. Intellectual function and level of education were relatively high, and there was less influence on cognitive function in comparison to earlier studies. Third, most probands had well-preserved global function. We recruited probands in an outpatient setting. A large portion of the probands maintained relatively good function. Some were married and had jobs. The relatives also functioned well. We examined individuals with higher levels of functioning in order to minimize confounding factors that can affect test performance in a non-specific manner. However, despite these advan- tages in cognitive function compared to previous studies, specific impairments were observed qualitatively and quantitatively in the different samples. Fourth, our samples were taken from a more homogenous population in terms of diagnosis and ethnicity. Samples were not restricted to a specific diagnosis in some previous studies, such as schizophrenia spectrum disorder or bipolar spectrum disorder. In addition, it is well known that Koreans are one "ethnic family", speaking one language.

There are some limitations in this study. First, the sample size may not be sufficient to represent the cognitive characteristics of probands and relatives. This is due to the strict selection criteria. Second, we attempted to cover a broad range of neuropsychological domains. However, we could not employ many test batteries because of some practical issues. Third, the effects of medication on cognitive function are unclear. All of the probands were taking psychiatric medication in this study. Benzodiazepine is one of the renowned drugs that causes cognitive impairment. ${ }^{50)}$ However, the influence of antipsychotic medication on cognitive functioning is not clear. ${ }^{51)}$ To adjust for the effect of drugs on cognition, the participation of drug-naïve probands could be encouraged, but there will be many obstacles in the course of recruiting drug naïve subjects, including ethical issues.

In conclusion, SZ-P showed the most generalized and severe cognitive dysfunction. Psychotic BD-P also revealed selective cognitive impairments, but less severe than those in SZ-P. The cognitive performance of both types of relative was intermediate to the performance of both types of probands and HCs. All of the proband and relative groups showed working memory dysfunction compared to the HC group. This finding suggests the working memory is a promising shared endophenotype between SZ and BD. A deficit in verbal fluency was observed only in SZ-P and SZ-R, which implies that verbal fluency may be an endophenotype for SZ specifically. The clinical expression of SZ and BD probably were related to impairments in verbal memory, visual memory, and executive function because they were not observed in family groups. These findings also support the notion that the two disorders may present a clinical continuum with overlapping cognitive characteristics. Further detailed studies should be conducted using a large number of subjects.

\section{- Acknowledgments}

This research was supported by Kyungpook National University Research Fund, 2012. 


\section{REFERENCES}

1. Keshavan MS, Morris DW, Sweeney JA, Pearlson G, Thaker G, Seidman LJ, et al. A dimensional approach to the psychosis spectrum between bipolar disorder and schizophrenia: the Schizo-Bipolar Scale. Schizophr Res 2011;133:250-254.

2. Arnone D, Cavanagh J, Gerber D, Lawrie SM, Ebmeier KP, McIntosh AM. Magnetic resonance imaging studies in bipolar disorder and schizophrenia: meta-analysis. $\mathrm{Br} J$ Psychiatry 2009;195:194-201.

3. De Peri L, Crescini A, Deste G, Fusar-Poli P, Sacchetti E, Vita A. Brain structural abnormalities at the onset of schizophrenia and bipolar disorder: a meta-analysis of controlled magnetic resonance imaging studies. Curr Pharm Des 2012;18:486-494.

4. Williams HJ, Norton N, Dwyer S, Moskvina V, Nikolov I, Carroll L, et al. Fine mapping of ZNF804A and genomewide significant evidence for its involvement in schizophrenia and bipolar disorder. Mol Psychiatry 2011;16: 429-441.

5. Lee KW, Woon PS, Teo YY, Sim K. Genome wide association studies (GWAS) and copy number variation $(C N V)$ studies of the major psychoses: what have we learnt? Neurosci Biobehav Rev 2012;36:556-571.

6. Gottesman II, Gould TD. The endophenotype concept in psychiatry: etymology and strategic intentions. Am J Psychiatry 2003;160:636-645.

7. Ivleva EI, Morris DW, Moates AF, Suppes T, Thaker GK, Tamminga CA. Genetics and intermediate phenotypes of the schizophrenia--bipolar disorder boundary. Neurosci Biobehav Rev 2010;34:897-921.

8. Hill SK, Harris MS, Herbener ES, Pavuluri M, Sweeney JA. Neurocognitive allied phenotypes for schizophrenia and bipolar disorder. Schizophr Bull 2008;34:743-759.

9. Vöhringer PA, Barroilhet SA, Amerio A, Reale ML, Alvear $\mathrm{K}$, Vergne $\mathrm{D}$, et al. Cognitive impairment in bipolar disorder and schizophrenia: a systematic review. Front Psychiatry 2013;4:87.

10. Sitskoorn MM, Aleman A, Ebisch SJ, Appels MC, Kahn RS. Cognitive deficits in relatives of patients with schizophrenia: a meta-analysis. Schizophr Res 2004;71:285-295.

11. Snitz BE, Macdonald AW 3rd, Carter CS. Cognitive deficits in unaffected first-degree relatives of schizophrenia patients: a meta-analytic review of putative endophenotypes. Schizophr Bull 2006;32:179-194.

12. Arts B, Jabben N, Krabbendam L, van Os J. Meta-analyses of cognitive functioning in euthymic bipolar patients and their first-degree relatives. Psychol Med 2008;38:771-785.

13. Balanzá-Martínez V, Rubio C, Selva-Vera G, Martinez-Aran A, Sánchez-Moreno J, Salazar-Fraile J, et al. Neurocognitive endophenotypes (endophenocognitypes) from studies of relatives of bipolar disorder subjects: a systematic review. Neurosci Biobehav Rev 2008;32:1426-1438.

14. Bora E, Yucel M, Pantelis C. Cognitive endophenotypes of bipolar disorder: a meta-analysis of neuropsychological deficits in euthymic patients and their first-degree relatives. $J$ Affect Disord 2009;113:1-20.

15. Glahn DC, Almasy L, Barguil M, Hare E, Peralta JM, Kent JW Jr, et al. Neurocognitive endophenotypes for bipolar disorder identified in multiplex multigenerational families. Arch Gen Psychiatry 2010;67:168-177.

16. Kéri S, Kelemen O, Benedek G, Janka Z. Different trait markers for schizophrenia and bipolar disorder: a neurocognitive approach. Psychol Med 2001;31:915-922.
17. Szöke A, Schürhoff F, Golmard JL, Alter C, Roy I, Méary $\mathrm{A}$, et al. Familial resemblance for executive functions in families of schizophrenic and bipolar patients. Psychiatry Res 2006;144:131-138.

18. Jabben N, Arts B, van Os J, Krabbendam L. Neurocognitive functioning as intermediary phenotype and predictor of psychosocial functioning across the psychosis continuum: studies in schizophrenia and bipolar disorder. J Clin Psychiatry 2010;71:764-774.

19. Ivleva EI, Morris DW, Osuji J, Moates AF, Carmody TJ, Thaker GK, et al. Cognitive endophenotypes of psychosis within dimension and diagnosis. Psychiatry Res 2012;196: 38-44.

20. Hill SK, Reilly JL, Keefe RS, Gold JM, Bishop JR, Gershon ES, et al. Neuropsychological impairments in schizophrenia and psychotic bipolar disorder: findings from the Bipolar-Schizophrenia Network on Intermediate Phenotypes (B-SNIP) study. Am J Psychiatry 2013;170:1275-1284.

21. American Psychiatric Association. Diagnostic and Statistical Manual of Mental Disorders. 4th ed. Washington, D.C.: American Psychiatric Association; 1994.

22. Hahn OS, Ahn JH, Song SH, Cho MJ, Kim JK, Bae JN, et al. Development of Korean version of structured clinical interview schedule for DSM-IV axis I disorder: interrater reliability. J Korean Neuropsychiatr Assoc 2000;39:362-372.

23. Ahn YM, Lee KY, Yi JS, Kang MH, Kim DH, Kim JL, et al. A validation study of the Korean-version of the Montgomery-Asberg depression rating scale. J Korean Neuropsychiatr Assoc 2005;44:466-476.

24. Jung HY, Cho HS, Joo YH, Shin HK, Yi JS, Hwang S, et al. A validation study of the Korean-version of the Young Mania Rating Scale. J Korean Neuropsychiatr Assoc 2003;42:263-269.

25. Chung YC, Cho GH, Park TW. Treatment response, remission and recovery in schizophrenia: Definition and criteria. Korean J Psychopharmacol 2006;17:5-12.

26. Silverstein A. Factor structure of the Wechsler Adult Intelligence Scale-Revised. J Consult Clin Psychol 1982;50: 661-664.

27. Yeom T, Park Y, Oh K, Lee Y. Korean version Wechsler adult intelligence scale. Seoul:Korean Guidance 4;1992. p.13-28.

28. Nuechterlein KH, Asarnow RF. Degraded Stimulus Continuous Performance Test (DS-CPT) Program for IBM-Compatible Microcomputers, Version 8.12. Los Angeles, CA:Nuechterlein and Asarnow;1999.

29. Kim H. A manual of Kims frontal-executive neuropsychological test. Daegu:Neuropsychology Press;2001.

30. Kang Y. Na D. Seoul neuropsychological screening battery (SNSB) manual. Seoul:Human Brain Research \& Consulting Co;2003.

31. Heaton RK. Wisconsin card sorting test: computer version 2. Odessa:Psychological Assessment Resources;1993.

32. Morrison MW, Gregory RJ, Paul JJ. Reliability of the Finger Tapping Test and a note on sex differences. Percept Mot Skills 1979;48:139-142.

33. Heinrichs RW, Zakzanis KK. Neurocognitive deficit in schizophrenia: a quantitative review of the evidence. Neuropsychology 1998;12:426-445.

34. Szöke A, Trandafir A, Dupont ME, Méary A, Schürhoff F, Leboyer M. Longitudinal studies of cognition in schizophrenia: meta-analysis. Br J Psychiatry 2008;192:248-257.

35. Glahn DC, Bearden CE, Cakir S, Barrett JA, Najt P, Serap Monkul E, et al. Differential working memory impairment in bipolar disorder and schizophrenia: effects of lifetime 
history of psychosis. Bipolar Disord 2006;8:117-123.

36. Glahn DC, Bearden CE, Barguil M, Barrett J, Reichenberg $\mathrm{A}$, Bowden $\mathrm{CL}$, et al. The neurocognitive signature of psychotic bipolar disorder. Biol Psychiatry 2007;62:910-916.

37. Martinez-Aran A, Torrent C, Tabares-Seisdedos R, Salamero M, Daban C, Balanza-Martinez V, et al. Neurocognitive impairment in bipolar patients with and without history of psychosis. J Clin Psychiatry 2008;69:233-239.

38. Kumar CT, Christodoulou T, Vyas NS, Kyriakopoulos M, Corrigall R, Reichenberg A, et al. Deficits in visual sustained attention differentiate genetic liability and disease expression for schizophrenia from Bipolar Disorder. Schizophr Res 2010;124:152-160.

39. Baddeley A. Working memory. Science 1992;255:556-559.

40. Park S, Püschel J, Sauter BH, Rentsch M, Hell D. Spatial working memory deficits and clinical symptoms in schizophrenia: a 4-month follow-up study. Biol Psychiatry 1999;46:392-400.

41. Heaton RK, Gladsjo JA, Palmer BW, Kuck J, Marcotte TD, Jeste DV. Stability and course of neuropsychological deficits in schizophrenia. Arch Gen Psychiatry 2001;58:24-32.

42. Tuulio-Henriksson A, Haukka J, Partonen T, Varilo T, Paunio $\mathrm{T}$, Ekelund $\mathrm{J}$, et al. Heritability and number of quantitative trait loci of neurocognitive functions in families with schizophrenia. Am J Med Genet 2002;114:483-490.

43. Bourne C, Aydemir Ö, Balanzá-Martínez V, Bora E, Brissos S, Cavanagh JT, et al. Neuropsychological testing of cognitive impairment in euthymic bipolar disorder: an individual patient data meta-analysis. Acta Psychiatr Scand 2013;128:149-162.

44. Curtis CE. Prefrontal and parietal contributions to spatial working memory. Neuroscience 2006;139:173-180.

45. Koch K, Wagner G, Nenadic I, Schachtzabel C, Schultz C, Roebel M, et al. Fronto-striatal hypoactivation during correct information retrieval in patients with schizophrenia: an fMRI study. Neuroscience 2008;153:54-62.

46. Meda SA, Bhattarai M, Morris NA, Astur RS, Calhoun VD, Mathalon DH, et al. An fMRI study of working memory in first-degree unaffected relatives of schizophrenia patients. Schizophr Res 2008;104:85-95.

47. Townsend J, Bookheimer SY, Foland-Ross LC, Sugar CA, Altshuler LL. fMRI abnormalities in dorsolateral prefrontal cortex during a working memory task in manic, euthymic and depressed bipolar subjects. Psychiatry Res 2010;182: 22-29.

48. Thermenos HW, Goldstein JM, Milanovic SM, WhitfieldGabrieli S, Makris N, Laviolette P, et al. An fMRI study of working memory in persons with bipolar disorder or at genetic risk for bipolar disorder. Am J Med Genet B Neuropsychiatr Genet 2010;153B:120-131.

49. Barch DM, Sheffield JM. Cognitive impairments in psychotic disorders: common mechanisms and measurement. World Psychiatry 2014;13:224-232.

50. Barker MJ, Greenwood KM, Jackson M, Crowe SF. Persistence of cognitive effects after withdrawal from long-term benzodiazepine use: a meta-analysis. Arch Clin Neuropsychol 2004;19:437-454.

51. Husa AP, Rannikko I, Moilanen J, Haapea M, Murray GK, Barnett $\mathrm{J}$, et al. Lifetime use of antipsychotic medication and its relation to change of verbal learning and memory in midlife schizophrenia - An observational 9-year follow-up study. Schizophr Res 2014;158:134-141. 